\title{
Information at Hand - Using Wearable Devices to Display Task Information in the Context of Industry 4.0
}

\author{
Sebastian $\operatorname{Mach}^{(\bowtie)}$, Almut Kastrau, and Franziska Schmalfuß \\ Chemnitz University of Technology, \\ Wilhelm-Raabe-Straße 43, 09120 Chemnitz, Germany \\ sebastian.mach@psychologie.tu-chemnitz.de
}

\begin{abstract}
In the context of Industry 4.0 and the extensive interconnection of every part of the production process, the industrial worker will still maintain a key role. It is important to support the workers by utilizing ongoing digitalization at work and also to provide suitable concepts for communication between human and automation. A possible solution could be the application of wearable devices such as smartwatches. They are able to automatically collect data about the state of the worker as well as provide relevant information for the worker about the status of machines. The aim of the present research is to investigate whether smartwatches are a suitable alternative to conventional methods of displaying information in an industrial environment - despite some limitations of smartwatches, like the small screen. Therefore, we conducted a laboratory study displaying different amount of information (a list of six tasks vs. only the next task) on different devices (monitor screen vs. smartwatch screen). We asked 32 participants to follow the displayed instructions and fulfill the tasks (crossword puzzles, Sudoku, number connection task). Afterwards, the participants rated the different types of information display with questionnaires (e.g., User Experience Questionnaire). Results show significant differences in some aspects of user experience supporting the advantage of utilizing smartwatches in an industrial context. However, information on a smartwatch should be as short and meaningful as possible.
\end{abstract}

Keywords: Industry $4.0 \cdot$ User experience $\cdot$ Smartwatch $\cdot$ Task information

\section{Introduction}

In times of Industry 4.0, every part of the production process becomes interconnected and the digitalization of the factory processes is steadily ongoing to increase the potential to respond individually to customer demands, achieve more flexibility, and use given resources efficiently [1]. The factory floor worker will still maintain an important role as most of the working tasks might shift more towards higher complexity, flexibility, and abstract problem solving. Therefore, one of the objectives of the developments in context of Industry 4.0 is to successfully support the worker in this complex and flexible work environment [2]. The research project Factory2Fit (funded by the European Union's Horizon 2020 program, grant agreement No. 723277) aims at 
developing automation solutions for the factory of the future, by placing the worker in the center. One technical approach is using wearable devices. They combine different functionalities by providing the opportunity to collect physiological data for detecting, for instance, high workload at work [3, 4] and by serving as an interface between human and automation. In detail, some wrist-worn devices, like smartwatches, provide the possibility to receive and display information [5]. As one example, the worker, who is not in sight distance to all machines, could get a message on a smartwatch when a machine stopped due to technical problems or if tool switching is necessary. Sending individual instructions and information location-independent to the worker via wearable devices has already been tested [6, 7]. Hao and Helo [6] showed that supporting industrial workers with individual information via wearable devices could increase the performance and quality of the work. Although the potential of presenting machine status information or up-coming tasks via smartwatches is undeniable, it has not been fully investigated which possibilities and limitations exist regarding how and to which extend the information should be displayed on such a size-reduced smartwatch screen.

In the presented study, we examined how task relevant information should be displayed on a smartwatch. The small screen restricts the possibilities to capture a greater amount of information [8]. Therefore, to conceive information quickly, the small screen should only show essential information [9]. Additionally, users of small screens value good quality information and prefer rather more than less information [10]. Nevertheless, simplicity of an interface improves the user experience, in particular usability [11]. Usability describes "the extent to which a product can be used by specified users to achieve specified goals with effectiveness, efficiency, and satisfaction in a specified context of use" [12]. According to Schrepp and colleagues [13], user experience covers a broader range of facets describing the interaction with a product; including usability criteria (e.g., efficiency, controllability or learnability), and hedonic quality criteria [14] (e.g., stimulation, fun-of-use, novelty, emotions [15] and aesthetics [16]). A good user experience is essential for a successful implementation of new HMIs in work environments and research how to design interfaces of wearable devices to fulfill this criterion is rare.

One typical usage context for displaying information at work is, for example, displaying information about work tasks. This can be realized by supplying a list of tasks from which the worker can choose the next task, or by presenting only the next task, the worker should accomplish. The first option provides a better overview about the upcoming work and presenting a list of tasks provides a larger scope of action and makes use of a greater flexibility [17]. However, the latter option is corresponding with the demand of displaying sparse information on a smartwatch.

Following these results, we hypothesize that displaying information on a smartwatch provides better user experience compared to monitor screen (H1). However, for a task list, the literature review does not allow the formulation of a hypothesis. It needs to be explored if the user experience with a smartwatch exceeds the user experience with a monitor screen when information load is higher.

Furthermore, the presented research allows the assumption that presenting a list of tasks enhances user experience compared to only presenting the next task (H2). With this investigation, we aim at recommendations concerning information display on wearable devices in an industrial context. 


\section{Method}

Thirty-two university students (17 female, 15 male) participated in a laboratory experiment. They were recruited via student mailing lists and received course credits for their participation. The average age was 24.7 years $(S D=4.16)$. Only three participants had experience with a smartwatch for more than one week, others had less or no experience $(n=29)$.

The experiment consisted of a 2 (information format) $\times 2$ (display format) factorial within-subject design and involved three different stations where participants had to perform varying tasks. The designated tasks were presented as an action item list ('task list') or by showing the 'next task' (information format) via smartwatch or monitor screen (display format). The order in which the different conditions had to be accomplished as well as the order of the three different tasks were randomized using the Latin square. The actual tasks, the participants were asked to perform, were neither relevant for the evaluation of the information format nor the display format. The tasks included crossword puzzles, Sudoku, and a number connection task, which took 1-3 min to complete each.

The study procedure was submitted to the ethical committee of the Chemnitz University of Technology and no objections were expressed (no. V-240-15-SMSmartwatch-27112017).

The facets of user experience were collected using the paper-pencil version of User Experience Questionnaire (UEQ) [18]. This questionnaire includes 26 items and consists of six subscales: attractiveness, perspicuity, efficiency, dependability, stimulation and novelty. The UEQ allows a quick assessment of the user experience and covers main aspects of usability (efficiency, perspicuity, and dependability) as well as user experience aspects (originality and stimulation). The participants rated their impressions on a seven-point semantic differential. All sub-scales showed satisfying internal reliabilities $\left(.871 \leq\right.$ Cronbachs $\alpha_{\text {Attractiveness }} \leq .927 ; .690 \leq$ Cronbachs $\alpha_{\text {Perspicuity }} \leq .895 ;$ $.658 \leq$ Cronbachs $\quad \alpha_{\text {Efficiency }} \leq .758 ; .602 \leq$ Cronbachs $\quad \alpha_{\text {Dependability }} \leq .832$; $.704 \leq$ Cronbachs $\alpha_{\text {Stimulation }}<.855 ; .727 \leq$ Cronbachs $\alpha_{\text {Novelty }} \leq .882$ ).

In the monitor condition, the list of tasks or the next task was displayed on a 22-inch Dell P2210 monitor screen. For presenting the list of tasks or the next task on the smartwatch, the Samsung Gear S3 Frontier was utilized. This device comes along with a self-developer kit, so that the possibility can be implemented to send messages via Bluetooth or WiFi independently from external, commercial services. Therefore, a special Universal Windows Platform application (UWP) as well as a Tizen application needed to be developed (see Fig. 1). Via the UWP application, the messages could be generated, and via the Tizen application, they could be received on the smartwatch.

The procedure was as follows. First, the participants read the participant information about the study and confirmed their consent. The three different tasks (crossword puzzles, Sudoku, and number connection task) were introduced by the experimenter. Afterwards, the smartwatch was put on the left arm by the participant. Depending on the condition, the participant was asked to either watch the monitor screen or the smartwatch for an instruction about the upcoming/actual task. If there were no more open questions, they passed through the four experimental conditions. 

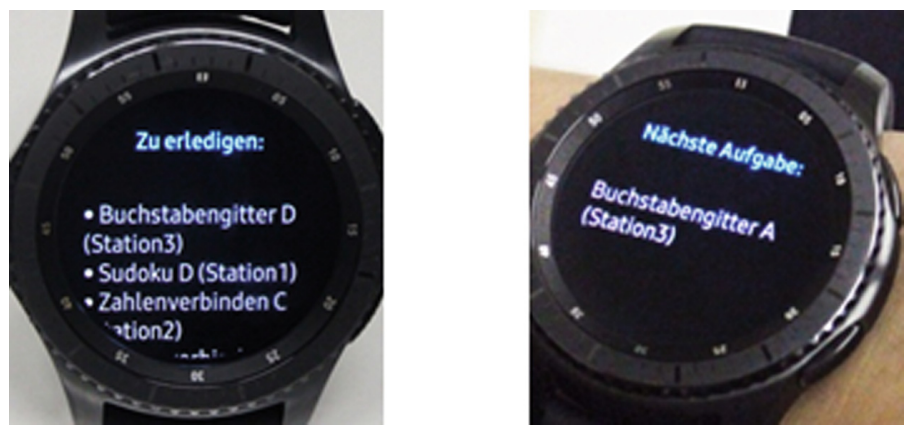

Fig. 1. List of tasks displayed on smartwatch (left, "To do: crossword puzzle D (station 3)...") or next task displayed on a smartwatch (right, "Next task: crossword puzzle A, (station 3)").

Within each condition, six tasks (each task twice) were processed. At the end of each condition, participants filled out the UEQ [18]. After all four conditions, they answered the demographic questionnaire. In the end, the participants could rate their favorite condition. In sum, the experiment lasted from 60 to $80 \mathrm{~min}$.

\section{Results}

The data from the UEQ was digitalized in a data matrix following the analysis instruction provided [19]. According to the authors of the UEQ, mean scores were transformed ( -3 to 3) [18]. The statistical description and calculations were done with RStudio [20]. For testing the hypotheses, an ANOVA with repeated measures was calculated [21] and assumptions were tested and fulfilled. The mean scores in each condition are shown in Fig. 2.

Results of the ANOVA with repeated measures regarding the display format supported partly hypothesis H1 (see Table 1). When comparing the display formats smartwatch and monitor screen, scores of the subscales attractiveness, stimulation, and novelty differed significantly. Compared to the monitor, participants rated the smartwatch as more attractive $\left(M_{\text {Smartwatch }}=1.03, S D=0.86 ; M_{\text {Monitor }}=0.14, S D=0.82\right)$ and were more stimulated to use the product $\left(M_{\text {Smartwatch }}=0.96, S D=0.82\right.$; $\left.M_{\text {Monitor }}=-0.41, S D=0.81\right)$. They also rated the novelty of the smartwatch significantly higher $\left(M_{\text {Smartwatch }}=1.07, S D=0.86\right)$ than the novelty of the monitor $\left(M_{\text {Monitor }}=-1.49, S D=0.87\right)$. According to Cohen [22], effects were large. Results displayed in Table 1 also show that the display format had no significant effects in perspicuity $\left(M_{\text {Smartwatch }}=1.57, S D=0.85 ; M_{\text {Monitor }}=1.63, S D=0.94\right)$, efficiency $\left(M_{\text {Smartwatch }}=1.25, \quad S D=0.83 ; \quad M_{\text {Monitor }}=1.04, \quad S D=0.85\right)$ and dependability $\left(M_{\text {Smartwatch }}=1.14, S D=0.97 ; M_{\text {Monitor }}=1.23, S D=0.75\right)$.

Hypothesis $\mathrm{H} 2$ was not supported by the data. Concerning the information format, the subscales attractiveness, perspicuity, efficiency, and stimulation differed highly significant (see Table 1), but the effect had the opposite direction. Contrary to our hypothesis, participants evaluated the information format, where only the next task was 


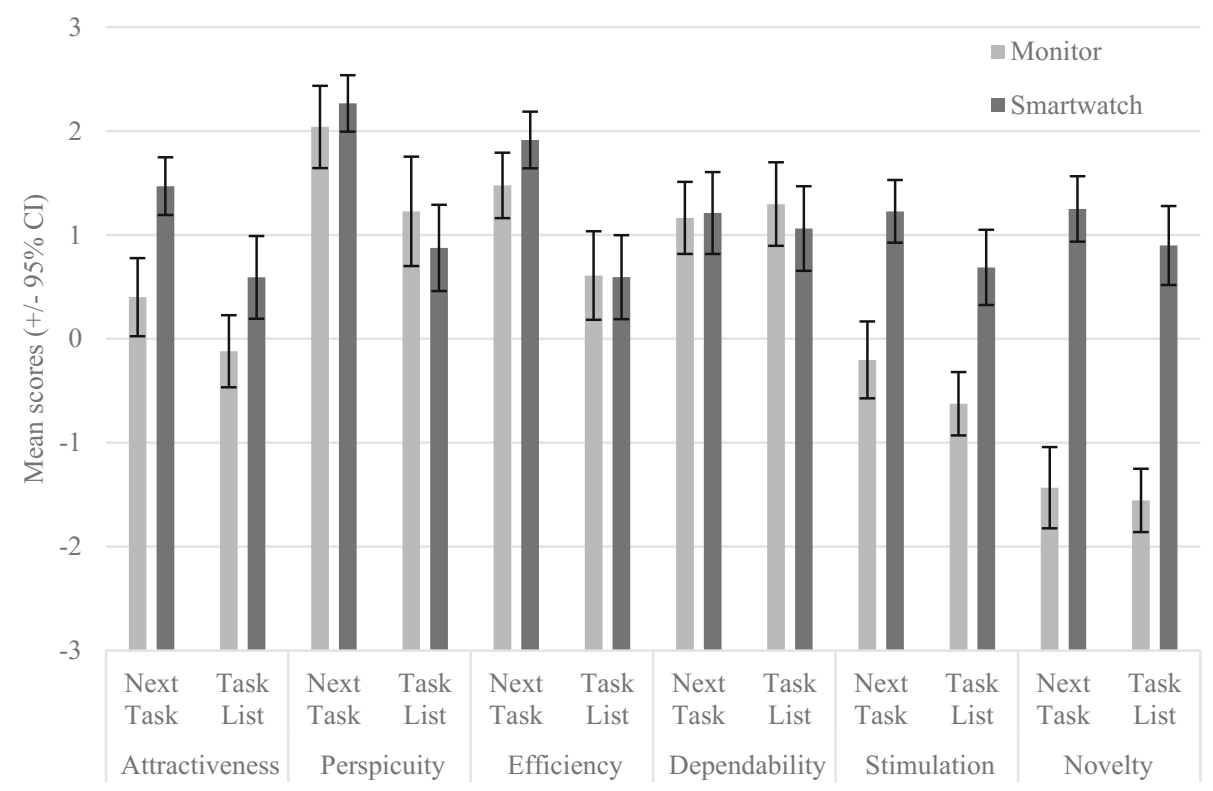

Fig. 2. Mean scores of UEQ subscales compared between the different experimental conditions.

Table 1. Results of ANOVAs with repeated measures for UEQ subscale scores.

\begin{tabular}{|c|c|c|c|c|c|c|c|c|c|}
\hline \multirow[t]{2}{*}{ Sub-scale } & \multicolumn{3}{|c|}{ Display format } & \multicolumn{3}{|c|}{ Information format } & \multicolumn{3}{|c|}{ Interaction } \\
\hline & $F$ & $(d f)$ & $\eta^{2}$ & $F$ & $(d f)$ & $\eta^{2}$ & $F$ & $(d f)$ & $\eta^{2}$ \\
\hline Attractiveness & $38.83^{* * *}$ & $(1,31)$ & .17 & $24.83^{* * * *}$ & $(1,31)$ & .11 & 2.53 & $(1,31)$ & .01 \\
\hline Perspicuity & 0.14 & $(1,31)$ & .00 & $44.87^{* * * *}$ & $(1,31)$ & .20 & $4.23^{*}$ & $(\mathbf{1 , 3 1})$ & .02 \\
\hline Efficiency & 1.46 & $(1,31)$ & .01 & $60.57^{* * *}$ & $(1,31)$ & .24 & 3.33 & $(1,31)$ & .01 \\
\hline Dependability & 0.50 & $(1,31)$ & .01 & 0.002 & $(1,31)$ & .00 & 1.46 & $(1,31)$ & .00 \\
\hline Stimulation & $55.13^{* * * *}$ & $(1,31)$ & .36 & $14.17^{* * * *}$ & $(\mathbf{1 , 3 1})$ & .06 & 0.37 & $(1,31)$ & .00 \\
\hline Novelty & $132.5^{* * * *}$ & $(1,31)$ & .64 & 3.44 & $(1,31)$ & .02 & 1.73 & $(1,31)$ & .00 \\
\hline
\end{tabular}

displayed, as more attractive (Attractiveness: $M_{\mathrm{List}}=0.24, S D=0.88 ; M_{\mathrm{Next}}=0.93$, $S D=0.77$ ), easier to get familiar with (Perspicuity: $M_{\text {List }}=1.05, S D=1.11$; $M_{\text {Next }}=2.15, S D=0.6$ ), more helping to solve the tasks (Efficiency: $M_{\text {List }}=0.60$, $\left.S D=0.94 ; M_{\mathrm{Next}}=1.70, S D=0.59\right)$, and more exciting and higher motivating (Stimulation: $M_{\text {List }}=0.03, S D=0.71 ; M_{\text {Next }}=0.51, S D=0.74$ ). The findings showed medium to large effects.

The only significant interaction between display format and information format occurred within the subscale perspicuity; participants got easier used to the presentation of only the next task when using the smartwatch, though the effect size was rather small. 


\section{Discussion}

The main aim of this study was to investigate the possibilities to display task relevant information on a smartwatch and provide recommendations for the application in an industrial environment.

Therefore, participants were presented with action items in form of a list of tasks or only the next task. The task or the list of tasks were displayed on a monitor screen or on a smartwatch. We hypothesized that compared to a monitor screen, displaying information on a smartwatch improves the user experience. In accordance with the hypothesis, participants rated the smartwatch as more attractive, were more excited and motivated to use the product, and perceived the smartwatch as innovative. Sending individual instructions and information location-independently [7] to the participants via smartwatch might be perceived as more flexible, and therefore more attractive. Also, these aspects of increased user experience might be partly explained by the composition of the sample, in which only three participants were familiar with a smartwatch and used it more than one week.

As for the second hypothesis, we assumed that a list of tasks enhances user experience compared to only presenting the next task. Opposite to our assumption, the participants rated the display format, where only the next task was displayed, as more attractive, easier to get familiar with, more helping to solve the tasks, and perceived the format as more exciting and motivating. This supports previous findings, that simplicity, especially the limited amount of information, enhances usability aspects like perspicuity and efficiency [11]. The increased information density that comes along with a task list seems to have not such a strong negative influence on the user experience as expected. This might be because of the experimental setting. Displaying other tasks, that have to be done in the future, did not provide important additional information. In another context, where the interconnection between the tasks and the sequence is important, displaying a list of tasks, from which the user can choose, might be more suitable.

The display format had no significant effects in perspicuity, efficiency, and dependability leading to the assumption, that these aspects are not as relevant for the evaluation of the devices. In addition, novelty might be not relevant for the information format.

In summary, untrained and novice persons see the new technology as exiting and supportive for displaying task relevant information. However, developers should aim at presenting information on a smartwatch as short and meaningful as possible. Future research should investigate the long-term effects of superiority concerning user experience of smartwatches. Therefore, industrial workers should be consulted, and the study should be replicated in an industrial context. In addition, a variation of display design and information format should be investigated.

In conclusion, the present study provided evidence that displaying information via a smartwatch can lead to increased user experience compared to a monitor. Additionally, the information should be as compact as possible but qualitatively adequate. 


\section{References}

1. MacDougall, W.: Industrie 4.0: Smart Manufacturing for the Future. Germany Trade \& Invest, Berlin (2014)

2. Kagermann, H., Wahlster, W., Helbig, J.: Securing the future of German manufacturing industry. In: Recommendations for Implementing the Strategic Initiative INDUSTRIE 4.0, Final Report of the Industrie 4.0 Working Group. Forschungsunion (2013)

3. Swan, M.: Sensor mania! The internet of things, wearable computing, objective metrics, and the quantified self 2.0. J. Sens. Actuator Netw. 1, 217-253 (2012)

4. Schmalfuß, F., Mach, S., Klüber, K., Habelt, B., Beggiato, M., Körner, A., Krems, J.F.: Potential of wearable devices for mental workload detection in different physiological activity conditions. In: de Waard, D., Di Nocera, F., Coelho, D., Edworthy, J., Brookhuis, K., Ferlazzo, F., Franke, T., Toffetti, A.: (eds.), Proceedings of the Human Factors and Ergonomics Society Europe Chapter (2017). http://hfes-europe.org

5. Zhang, Y., Rau, P.L.P.: Playing with multiple wearable devices: exploring the influence of display, motion and gender. Comput. Hum. Behav. 50, 148-158 (2015)

6. Hao, Y., Helo, P.: The role of wearable devices in meeting the needs of cloud manufacturing: a case study. Robot. Comput.-Integr. Manuf. 45, 168-179 (2015)

7. Jo, H., Kang, S., Kwon, H.J., Lee, J.D.: In-door location-based smart factory cloud platform supporting device-to-device self-collaboration. In: 2017 IEEE International Conference Big Data and Smart Computing (BigComp), pp. 348-351 (2017)

8. Jones, M., Marsden, G., Mohd-Nasir, N., Boone, K., Buchanan, G.: Improving web interaction on small displays. Comput. Netw. 31(11-16), 1129-1137 (1999)

9. Budiu, R.: The Apple Watch: User-Experience Appraisal. https://www.nngroup.com/ articles/smartwatch/. Accessed 29 Mar 2018

10. Jones, M., Buchanan, G., Thimbleby, H.: Sorting out searching on small screen devices. In: Paternò, F. (ed.) Mobile HCI 2002. LNCS, vol. 2411, pp. 81-94. Springer, Heidelberg (2002). https://doi.org/10.1007/3-540-45756-9_8

11. Lee, D., Moon, J., Kim, Y.J., Mun, Y.Y.: Antecedents and consequences of mobile phone usability: linking simplicity and interactivity to satisfaction, trust, and brand loyalty. Inf. Manag. 52(3), 295-304 (2015)

12. ISO: ISO 9241-210. Human-Centred Design for Interactive Systems. International Standard. International Organization for Standardization (2010)

13. Schrepp, M., Hinderks, A., Thomaschewski, J.: Applying the user experience questionnaire (UEQ) in different evaluation scenarios. In: Marcus, A. (ed.) DUXU 2014. LNCS, vol. 8517, pp. 383-392. Springer, Cham (2014). https://doi.org/10.1007/978-3-319-07668-3_37

14. Hassenzahl, M.: The effect of perceived hedonic quality on product appealingness. Int. J. Hum.-Comput. Interac. 13, 479-497 (2001)

15. Norman, D.: Emotional Design: Why We Love (Or Hate) Everyday Things. Basic Books, Boulder (2003)

16. Tractinsky, N.: Aesthetics and apparent usability: empirical assessing cultural and methodological issues. In: CHI 1997 Electronic Publications (1997). http://www.acm.org/ sigchi/chi97/proceedings/paper/nt.htm

17. Gustavsson, T.K., Jerbrant, A.: Task lists as infrastructure: an empirical study of multi-project work. Int. J. Proj. Organ. Manag. 4(3), 272 (2012)

18. Laugwitz, B., Held, T., Schrepp, M.: Construction and evaluation of a user experience questionnaire. In: Holzinger, A. (ed.) USAB 2008. LNCS, vol. 5298, pp. 63-76. Springer, Heidelberg (2008). https://doi.org/10.1007/978-3-540-89350-9_6

19. UEQ-Online Homepage. http://www.ueq-online.org/. Accessed 29 Mar 2018 
20. RStudio Team: RStudio: Integrated Development for R. RStudio Inc., Boston MA (2016). http://www.rstudio.com/

21. Lawrence, M.A.: ez: easy analysis and visualization of factorial experiments. R package version 4.4-0 (2016). https://CRAN.R-project.org/package=ez

22. Cohen, J.: Statistical Power Analysis for the Behavioral Sciences. Lawrence Earlbaum Associates, Hilsdale (1988)

Open Access This chapter is licensed under the terms of the Creative Commons Attribution 4.0 International License (http://creativecommons.org/licenses/by/4.0/), which permits use, sharing, adaptation, distribution and reproduction in any medium or format, as long as you give appropriate credit to the original author(s) and the source, provide a link to the Creative Commons license and indicate if changes were made.

The images or other third party material in this chapter are included in the chapter's Creative Commons license, unless indicated otherwise in a credit line to the material. If material is not included in the chapter's Creative Commons license and your intended use is not permitted by statutory regulation or exceeds the permitted use, you will need to obtain permission directly from the copyright holder.

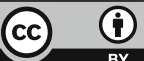

